\title{
Imagery and rehearsal as study strategies for written or orally presented passages
}

\author{
ROSSANA DE BENI and ANGELICA MOÈ \\ University of Padua, Padua, Italy
}

\begin{abstract}
In the present research, we studied the influence of text presentation modality on recallunder imagery, rehearsal, and no strategy instructions. It was hypothesized that imagery is a more effectiverecall strategy for an oral presentation and rehearsal for a written presentation, and that imagery and rehearsal are effective study strategies. One hundred twenty participants participated, 80 of whom were trained in the use of imagery or rehearsal in the study of passages and 40 of whom made up the control group. A text was presented orally or in writing; the trained participants were to memorize it using the strategy taught, and the participants in the control group were to memorize it using a freely chosen strategy. They were then asked for free written recall. The results confirmed that the participants using imagery recalled the oral presentation better than the written one, and those using rehearsal recalled the written presentation better than the oral one. The discussion focuses on a selective-interference explanation of the presentation modality effects. Practical suggestions are given, and implications for future research are discussed.
\end{abstract}

Imagery and rehearsal strategies are very frequently used during reading or study of a text (see, e.g., Richardson, 1998). Their spontaneous use increases with age (Schneider \& Sodian, 1997) and education (Soler \& Ruiz, 1996), being more frequent in college than in highschool students, and suggests that they are considered to be effective in improving comprehension and recall. This idea has been confirmed in a great number of studies that have shown that the use of good and well-elaborated images (see, e.g., Paivio, 1995) and the correct rehearsal of cue words (see, e.g., Gardiner, Gawlick, \& RichardsonKlavehn, 1994; Rodríguez \& Sadoski, 2000) lead to superior memory performance involving narrative selections. Efficacy is even greater when both imagery and rehearsal strategies are used simultaneously (Kalyuga, Chandler, \& Sweller, 1999; Plass, Chun, Mayer, \& Leutner, 1998). When used separately, imagery is generally considered more effective than rehearsal with unrelated material. Nevertheless, given particular conditions (e.g., time constraints), rehearsal may be more effective than imagery (van Hell \& Mahn, 1997). With passages, Simpson, Olejnik, Tam, and Supattathum (1994) found that elaborative rehearsal is an effective study strategy and that the quality of the elaborations produced is a very good predictor of academic achievement. Cornoldi and De Beni (1991) found that the loci method, the efficacy of which is based on both imagery and spatial components, is more useful than rehearsal for memorizing an

We thank Sandra De Carli, Simona Gardini, and Francesca Calzavara for collecting data and three anonymous referees for their helpful suggestions. Correspondence concerning this article should be addressed to R. De Beni, Department of General Psychology, University of Padua, Via Venezia, 8, 35131 Padua, Italy (e-mail: rossana.debeni@unipd.it). orally presented text, but not for memorizing a text presented in writing. De Beni, Moè, and Cornoldi (1997) demonstrated that the reduced efficacy of the loci method in written presentation depends on a selective interference effect between reading and imagery. The modality principle, a similar facilitating effect of oral presentation, has been found in multimedia learning with the use of computer-based instructional material consisting of texts presented orally as speech rather than visually as text, and concurrent on-screen animation (Mayer \& Moreno, 1998, 2002). This suggests that the use and efficacy of imagery and rehearsal may depend on presentation modality.

The selective interference theory predicts a disruption in performance when two tasks are performed by means of the same system (i.e., visual or auditory), but not when they are performed using different systems. Brooks $(1967,1968)$ found that mental visualization of a matrix is disrupted when subjects read its description (as in a concurrent visual task), but not when they listen to it (as in a concurrent auditory task). In contrast, when subjects are required to repeat material rather than visualize it, reading is a more effective means of presentation than listening. Baddeley and Lieberman (1980) found that a visuospatial task disrupts memory performance when subjects use imagery-based strategies, but not when verbal strategies are employed. Logie (1986) showed that unexpected visual material disrupts the memory performance of subjects using an imagery-based strategy, and unexpected speech disrupts the memory performance of subjects using rehearsal. This interfering effect of visual activity on imagery is confirmed by research carried out in the neuro- and psychophysiological fields. The imagination and visual perception have common neural substrates, so the same regions involved in vision are used in 
imagining (Farah, 1988). Subjects affected by cortical blindness are not able to use mental images, although other cognitive abilities are preserved (Roland \& Friberg, 1985).

Selective interference depends on the fact that humans have separate information-processing systems for visual and auditory information-namely, the phonological loop for speech-based information and the visuospatial sketch pad for visually and spatially based information. Each of these systems is limited in capacity (Baddeley, 1986). Visually presented information is processed-at least initially $\longrightarrow$ on the visuospatial sketch pad. Information presented auditorily is processed-at least initially - in the phonological loop. Sweller, van Merriënboer, and Paas (1998) demonstrated that if the same (visual or auditory) system is used to process information, longer times are needed to shift from one source of information to another. This shifting has been called the split-attention effect and is due to overcharging the same system (e.g., the visuospatial sketch pad for text and diagrams presented simultaneously, or the phonological loop for simultaneous sounds and narration). In the absence of shifting (i.e., when both visual and auditory systems are used), the effective capacity of working memory can be increased and performance maximized. Moreno and Mayer (1999) found that when words are held in the phonological loop (auditory presentation) and pictures in the visuospatial sketch pad (visual presentation), subjects are able to devote more attention to comprehending the text being processed.

The first aim of the present research was to determine whether there is an oral presentation effect - that is, a facilitation of the oral presentation modality-when a passage is studied with a simple, imagery-based strategy. It was predicted that participants instructed to image the cue words of the passage would recall an oral presentation better than a written presentation because of the absence of selective interference between encoding systems. The second aim was to determine whether there is a written presentation effect (i.e., a facilitating effect of written presentation) for participants using rehearsal (i.e., those instructed to repeat the cue words of the passage). If the oral presentation effect depends on the absence of selective interference between listening and imagining, a written presentation effect could be expected to exist between reading and rehearsing. To this end, the performance of a group instructed in the use of imagery was compared with that of a group instructed in rehearsal during study of a passage presented either orally or in written form. Imagery and rehearsal are similar in complexity, but whereas imagery is a visual strategy, rehearsal is verbal. Moreover, a control group with no strategy training was created to ensure that the two presentation modalities were equally difficult and to verify the effectiveness of imagery and rehearsal strategies in the study of passages.

In natural situations, the written and oral modalities differ in many aspects, one of which is whether the visual or the auditory system is involved. The written presentation, to which participants are accustomed, is on paper. This differs from the oral modality also in that it permits the use of strategies linked to self-pacing, such as returning to previously read parts of the texts, stopping on the important points, and reading difficult paragraphs of different levels of difficulty at different speeds. In general, none of these strategies can be activated for an oral presentation. Therefore, to compare oral and written presentations correctly, we created a controlled written presentation on a computer using a moving-window modality (see the Procedure section for details). This modality appears more appropriate than the presentation of words at the same point at the center of a screen, in that it creates a reading situation that allows movement of gaze from left to right, as in a natural reading situation (Kieras \& Just, 1984). The oral modality included the presentation of material with the use of a tape recorder.

The hypotheses were that (1) participants using imagery (i.e., those instructed to image the selected cue words of the passage) would recall more of an oral presentation (oral presentation effect) owing to the absence of selective interference between imaging and listening, (2) participants using rehearsal (i.e., those instructed to repeat the selected cue words of the passage) would be helped more by written than by oral presentation of material (written presentation effect) because use of the absence of selective interference between rehearsing and reading, and (3) imagery and rehearsal would be effective study strategies - that is, participants instructed to select cue words of a passage and to image or repeat them would recall more than would the no-strategytraining group left free to use a strategy of their own choosing.

\section{METHOD}

\section{Participants and Design}

One hundred twenty first-year Italian psychology students (27 males and 93 females) took part in this experiment. The mean age was 21.32 years (ranging from 19 to 27 years). The design was 3 groups (imagery vs. rehearsal vs. control) $\times 2$ presentation modalities (oral vs. written).

\section{Materials}

We used three different passages in Italian: two for the training session and one for the experimental session. For the training session, one passage (entitled "The Wildcat") consisted of 253 words and dealt with the characteristics and life habits of the wildcat, and the other (entitled "The Birch") consisted of 265 words and dealt with the characteristics and practical uses of the birch tree. The passage for the experimental training was on the history of Sudan (entitled "History of Sudan") and consisted of 401 words. The topics of all three passages were chosen from those least well known to all the participants, with the aim of avoiding a facilitating effect due to previous knowledge of the material to be studied. A 20 -item word list was used for the training phase.

\section{Procedure}

The participants were randomly divided into an imagery group, a rehearsal group, and a control group, depending on the kind of 
training administered, with 40 participants per group. The three groups were equivalent for mean age and male/female proportion. Half of the participants in each group were given the oral presentation and the other half the written presentation.

Phase 1: Training. The day before the experimental session, the participants in the imagery and rehearsal groups were instructed in the use of imagery or rehearsal for studying passages during a 2-h training session in small groups of about 10 participants each.

First, the participants in the imagery group were taught how to create effective images of concrete and abstract words using the characteristics of vividness, interactivity, personal reference, specificity, and so on (see, e.g., Denis, 1995). The participants in the rehearsal group were shown how to correctly use mental rehearsalthat is, how to verbally repeat. A 20 -item word list was used in the exercise, and the participants were asked to say which image or which repetition they made for each term.

Second, both groups were taught the cue-word method, practicing with the passage "The Wildcat." The participants were instructed in how to (1) select a cue word (i.e., the one most representative of the meaning of a paragraph or group of sentences) — for instance, for the sentence, "The wildcat is a very rare animal," they could select the cue word "rare"; and (2) create an image or verbalization of the cue word-for instance, to visualize just one cat in the middle of a landscape or repeat the word rare. During retrieval, the participants were asked to recall the imagined or verbalized cue words and, from them, the content of the passage. The participants were asked to read a paragraph, select and write down one or more cue words, and then image or repeat the cue words. They were then asked to give free written recall. This exercise was carried out very slowly in a group discussion climate. Both groups were then invited to practice selecting the cue words and to imagine or rehearse them with the passage "The Birch," individually and within a limited time, to simulate a real study situation. They were then asked for free recall. Finally, time was allowed for group discussion with the aim of resolving any participants' doubts.

Phase 2: Experimental session. The day after the training session (any day for the control group), the participants came to the laboratory individually for the experimental session. Those in the imagery and rehearsal groups were instructed to memorize the passage "History of Sudan" using the specific method learned. The participants in the control group were instructed to use any strategy they wished. They were told they would be required to recall the passage at the end of the presentation and write the main concepts on a sheet of paper. The following is a translation of the Italian instructions to the participants:

You will be presented a passage. Listen to (read) it carefully and try to memorize it using imagery (or rehearsal or any strategy). At the end of the passage you will be asked to recall the content by writing down the main concepts.

The passage was presented either by tape recorder (oral presentation) or computer (written presentation). Using the MEL system (Schneider, 1988) and a mowing-window modality, the computer displayed one to three words at a time (maximum 15 characters), running in a line along the screen for $900 \mathrm{msec}$. The participants had to form their images (or rehearse or use any strategy of their choosing) while the words were being presented. No additional time was allowed at the end of the presentation. Both oral and written presentations lasted $4 \mathrm{~min}$. After a 1-min interpolated task (i.e., counting backwards from a three-digit number), the participants were required to write down the main concepts of the passage, maintaining the presentation order as well as possible. A maximum of $10 \mathrm{~min}$ was allowed. Finally, they were asked about their previous knowledge of the passage. All the participants declared that the topic and information presented were completely new to them.

Phase 3: Final interview. For the imagery and rehearsal groups, the participants were asked to answer three questions written on a piece of paper, to check whether they had followed the instructions. The questions were as follows: (1) How many images (or repetitions) did you make? (The participants had to answer using a 5-point Likert scale on which 1 corresponded to $a$ few and 5 to $a$ lot.) (2) How good were your images (or repetitions)? (The participants had to answer using a 5-point Likert scale on which 1 corresponded to bad and 5 to excellent. ) (3) Did you use any other strategies apart from imagery or rehearsal? (The possible responses were yes and no.) The participants in the control group were asked which strategies they had used to study the passage. Using a 5-point Likert scale, they had to rate the use of the following 10 strategies: mental repetition, concentration, imagination, mental maps or schemas, visualization as in a film, attention, links with previous knowledge, organization, selection of cue words, and relaxation.

\section{Scoring}

The passage was scored according to the method of De Beni et al. (1997). Two independent judges had previously divided the passage into 43 idea units, each corresponding to a concept (see the Appendix for an example). A third judge resolved the few disagreements. The same two independent judges then considered each idea unit recalled and allotted three points if the idea unit was both correctly recalled and in the right order, two points if the idea unit was either correctly recalled but not in the right order or incorrectly recalled but in the right order, and one point if the idea unit was incorrectly recalled and in an incorrect order. The third judge resolved the few disagreements. Partial scores were then summed.

For example, for the idea unit "One of the oldest kingdoms of the Black population was based in Sudan," three points were given if the recall was almost verbatim and this idea unit was recalled first; two points were allotted if the idea unit was recalled almost verbatim but not first, or if the idea unit was recalled first but some information was missing-for example, if the participant recalled that Sudan was a very old kingdom but not that the population was Black, or recalled that the population was Black but not that Sudan was one of the oldest kingdoms. Finally, one point was given if the idea unit was not recalled completely and not as the first.

Given that the passage contained 43 idea units and the highest score for each was 3 , the maximum theoretical score was 129 . The maximum score actually reached by a participant was 74 , corresponding to a $57 \%$ recall of the passage.

\section{RESULTS}

\section{Strategies Used by the Control Group}

A factor analysis was run on the rated use of the 10 different strategies available to the control group. The following three factors were extracted: imagery-based strategies (imagination, visualization as in a film, and relaxation), verbally based strategies (mental maps or schemas, organization, and selection of cue words), and concentration-based strategies (mental repetition, concentration, attention, and links with previous knowledge).

The explained variances were 20.24, 15.84, and 15.46 for the three factors. Mean rated use was $2.57(S D=.84)$, $2.47(S D=.66)$, and $3.00(S D=.51)$ for the imagerybased, verbally based, and concentration-based strategies, respectively. There was a significant difference between concentration-based and both imagery-based $[t(39)=2.66, p=.011]$ and verbally based $[t(39)=4.02$, $p<.001]$ strategies, which did not differ from each other. Only the rated use of concentration-based strategies correlated with mean recall $(r=.344, p=.030)$. 
This means that the participants in the control group did not systematically use either imagery-based or verbally based strategies and that they relied primarily on attention and concentration in listening to or reading the passage.

\section{Use of Imagery and Rehearsal by the Two Experimental Groups}

Some preliminary analyses were carried out to check whether (1) the participants in the two experimental groups had followed the instructions-that is, that they had used imagery or rehearsal and not other strategies (see the third question of the final interview) and (2) both groups had used the strategy taught (imagery or rehearsal) to the same extent (see the first and second questions of the final interview). To the third question, all the participants of both experimental groups answered "no,"-that is, they declared that they had not used strategies other than imagery or rehearsal (according to group). For the first and second questions, four separate chi-square analyses showed that there was no difference due to group or to presentation modality in the declared use and goodness of imagery and rehearsal. This means that, independently of the strategy taught and the presentation modality, the participants in the two experimental groups declared similar degrees of adherence to the specific strategy taught.

Only a minority declared that they had used only a few images or repetitions $(10 \%)$ or that their images or repetitions were not very good (9\%). Similarly low percentages can be observed for the highest scores: $6 \%$ and 5\% for the first and second questions, respectively. The majority of the participants declared moderate use of mental imagery or rehearsal (31\% answered "some," $40 \%$ "quite a lot," and 13\% "many"). Equally prudent were their evaluations of the quality of the images and repetitions produced (34\% judged them as "bad," $40 \%$ as "average," and $12 \%$ as "very good"). It is interesting that this is just a general tendency, probably owing to experimental constraints. The participants had to study an unknown passage for only 4 min and apply a specific strategy taught just the day before. Moreover, given that the trained participants averaged $27 \%$ on correct recall (mean recall was 35.10 on a maximum of 129), they may have misattributed their rather low recall scores to ineffective application of encoding strategies.

\section{Effects of Presentation Modality}

Given that the trained groups were identical in their declared use of imagery or rehearsal, a 3 (groups) $\times 2$ (presentations) ANOVA with both between-participants measures was carried out on the recall scores of the "History of Sudan" passage. It revealed a main effect of group $[F(2,114)=14.89, p<.001 ;$ mean recall was lower for the control group than for the imagery and rehearsal groups, which did not differ from each other] and a group $\times$ presentation interaction $[F(2,56)=17.60, p<$ .001 ; see Table 1].
Table 1

Mean Correct Recall for

Oral and Written Presentations by the Three Groups

\begin{tabular}{|c|c|c|c|c|c|c|}
\hline \multirow[b]{3}{*}{ Group } & \multicolumn{6}{|c|}{ Presentation Modality } \\
\hline & \multicolumn{2}{|c|}{ Oral } & \multicolumn{2}{|c|}{ Written } & \multicolumn{2}{|c|}{ Total } \\
\hline & $M$ & $S D$ & $M$ & $S D$ & $M$ & $S D$ \\
\hline Imagery & 42.95 & 10.56 & 33.25 & 9.61 & 38.10 & 11.11 \\
\hline Rehearsal & 24.15 & 10.50 & 40.05 & 11.35 & 32.10 & 13.46 \\
\hline Control & 28.10 & 9.60 & 23.00 & 10.00 & 25.55 & 10.01 \\
\hline
\end{tabular}

Note-Maximum possible score $=129$.

Post hoc comparisons using the Tukey test $(p<.05)$ showed a critical value of 9.43 . The imagery group recalled more of the oral than of the written presentation. The rehearsal group recalled more of the written than of the oral presentation. For the control group, there was no presentation modality effect. For the oral presentation, the imagery group recalled more than the other two groups, which did not differ from each other. For the written presentation, the control group recalled less than the other two groups, which did not differ from each other.

\section{DISCUSSION AND CONCLUSIONS}

Presentation modality effects were explored in participants using imagery or rehearsal and in a control group. Two groups were instructed to select and image or repeat the cue words of the passage to be studied. They and a control group were presented a passage to study and recall, either orally or in a controlled written presentation modality. As was hypothesized, the results confirmed a facilitating effect of oral presentation for participants using imagery and of written presentation for participants using rehearsal. For the control group, recall was the same in the written and oral presentations, thus confirming the equal difficulty of the two presentation modalities. Both imagery and rehearsal groups recalled more than the control group. Control group recall correlated only with concentration and not with the use of either imagery-based or verbally based strategies.

The occurrence of presentation modality effects could depend on selective interference or reading characteristics. Reading requires participants to deal with information in a more verbal form than listening, because of a phonological translation prior to or along with extraction of propositional representations (Coltheart, 1981). According to this view, reading requires both visual and phonological resources. Visual resources are needed to compare the visual characteristics of the word with the stored lexical information: The phonological component translates graphemes into phonemes. This phonological translation seems to be important for children who are learning to read, but it is rarely used by adults (i.e., in reading new words or nonwords; Frith, 1985). Adults do not regularly translate from grapheme to phoneme but read words they already know by direct access to the phonological form of the word-that is, without re- 
hearsing. Selective interference, rather than reading characteristics, therefore seems to be the best explanation for the presentation modality effects.

The two presentation modalities were made similar by preventing the participants from engaging in self-paced reading. Thus, it is possible to conclude that presentation modality effects are due to the involvement of two separate systems (a facilitating effect) or of the same system (an interfering effect). For participants using imagery, there is a facilitating effect when the auditory system is involved during encoding (oral presentation), since the imagery activity requires mainly the visual system; an interfering effect is found when the same visual system used to image is employed to read (i.e., with written presentation). On the contrary, for participants using rehearsal there is a facilitating effect when the visual system is involved during encoding (written presentation), since rehearsal requires mainly the verbal system; an interfering effect is found when the same verbal system used to repeat is employed for listening (oral presentation).

These facilitating or interfering effects of presentation modality on the use and efficacy of imagery or rehearsal strategies have been found in a very controlled situation. In natural situations, the interfering effect of reading on imagination is probably not manifested as damage to memory but as an extension of study time. In fact, to be able to use imagery strategies during reading, participants may need to look around and shift their glance from the text so that the visual input does not slow down the imaginative process (see, e.g., Glenberg, Schroeder, $\&$ Robertson, 1998). However, for the oral presentation, in real situations it is more difficult to modify listening time, for example by stopping the audio portion of a documentary or asking a speaker to reduce his or her speed.

The results of the experiment may suggest activation of verbally based strategies during study of a written text and activation of imagery-based strategies when one is listening to a discourse not accompanied by visual or figurative material. Actually, in these situations there is no selective interference between the systems used (auditory and visual) and the processes activated for use of the imagery-based or verbally based strategies. The best situation could be the simultaneous presentation of material in both oral and written modalities, such as during a lesson accompanied by a slide presentation, navigation on the Internet, or a video-recorded document. In these situations, both visual and auditory inputs are available at the same time. Participants are advantaged because they can encode using the modality that interferes less with the strategies used, preferring listening with imagerybased strategies and reading with verbally based strategies.

These facilitating or interfering effects of presentation modality have been found in a very restricted situation and occur in the encoding phase only. Future research is needed to extend the results obtained to different kinds of passages (e.g., passages that are easy or difficult to image, longer or shorter, or with or without illustrations) and to explore effects due to recall modality. We required the participants to give written recall only. In fact, the participants were required to recollect their images or repetitions during retrieval. It is possible to expect that the performance of participants using imagery could be facilitated by an oral recall modality and that of participants using rehearsal by a written recall modality. Congruence effects between presentation and recall modalities could also be expected.

\section{REFERENCES}

BADdeley, A. D. (1986). Working memory. Oxford: Oxford University Press, Clarendon Press.

Baddeley, A. D., \& Lieberman, K. (1980). Spatial working memory. In R. S. Nickerson (Ed.), Attention and performance VIII (pp. 521539). Hillsdale, NJ: Erlbaum.

BROOKS, L. R. (1967). The suppression of visualization by reading. Quarterly Journal of Experimental Psychology, 19, 289-299.

BRooKs, L. R. (1968). Spatial and verbal components of the act of recall. Canadian Journal of Psychology, 22, 349-368.

COlTHEART, M. (1981). Disorders of reading and their implications for models of normal reading. Visible Language, 15, 245-286.

Cornoldi, C., \& De Beni, R. (1991). Memory for discourse: Loci mnemonics and the oral presentation effect. Applied Cognitive Psychology, 5, 511-518.

DE Beni, R., MoÈ, A., \& CoRnOldi, C. (1997). Learning from texts or lectures: Loci mnemonics can interfere with reading but not with listening. European Journal of Cognitive Psychology, 9, 401-415.

DENIS, M. (1995). Vividness of visual imagery and the evaluation of its effects on cognitive performance. Journal of Mental Imagery, 19, 136-138.

FARAH, M. J. (1988). Is visual imagery really visual? Overlooked evidence from neuropsychology. Psychological Review, 95, 307-317. FrITH, U. (1985). Beneath the surface of developmental dyslexia. In J. C. Marshall, M. Coltheart, \& K. Patterson (Eds.), Surface dyslexia and surface dysgraphia (pp. 124-138). London: Routledge \& Kegan Paul.

GARDINER, J. M., GAWLICK, B., \& RichARDSON-KLAVEHN, A. (1994). Maintenance rehearsal affects knowing, not remembering; elaborative rehearsal affects remembering, not knowing. Psychonomic Bulletin \& Review, 1, 107-110.

Glenberg, A. M., Schroeder, J. L., \& Robertson, D. A. (1998). Averting the gaze disengages the environment and facilitates remembering. Memory \& Cognition, 26, 651-658.

Kalyuga, S., Chandler, P., \& Sweller, J. (1999). Managing splitattention and redundancy in multimedia instruction. Applied Cognitive Psychology, 13, 351-371.

KIERAS, D. E., \& JUST, A. M. (EDS.) (1984). New methods in reading comprehension research. Hillsdale, NJ: Erlbaum.

LogIE, R. H. (1986). Visuo-spatial processing in working memory. Quarterly Journal of Experimental Psychology, 38A, 229-247.

MAYER, R. E., \& MOREnO, R. (1998). A split-attention effect in multimedia learning: Evidence for dual processing systems in working memory. Journal of Educational Psychology, 90, 312-320.

Mayer, R. E., \& Moreno, R. (2002). Aids to computer-based multimedia learning. Learning \& Instruction, 12, 107-119.

MOREnO, R., \& MAYER, R. E. (1999). Cognitive principles of multimedia learning: The role of modality and contiguity. Journal of Educational Psychology, 91, 358-368.

PAIVIO, A. (1995). Imagery and memory. In M. S. Gazzaniga (Ed.), The cognitive neurosciences (pp. 977-986). Cambridge, MA: MIT Press.

Plass, J. L., Chun, D. M., MAYer, R. E., \& Leutner, D. (1998). Supporting visual and verbal learning preferences in a second-language multimedia learning environment. Journal of Educational Psychology, 90, 25-36.

RichARDSON, J. T. E. (1998). The availability and effectiveness of reported mediators in associative learning: A historical review and an experimental investigation. Psychonomic Bulletin \& Review, 5, 597-614. 
RodríGue Z, M., \& SADOSKI, M. (2000). Effects of rote, context, keyword, and context/keyword methods on retention of vocabulary in EFL classrooms. Language Learning, 50, 385-412.

ROLAND, P. E., \& FRIBERG, L. (1985). Localization of cortical areas activated by thinking. Journal of Neuropsychology, 53, 1219-1243.

SCHNEIDER, W. (1988). Micro Experimental Laboratory: An integrated system for IBM PC compatibles. Behavior Research Methods, Instruments, \& Computers, 20, 206-217.

SCHNEIDER, W., \& SodiAn, B. (1997). Memory strategy development: Lessons from longitudinal research. Developmental Review, 17, 442 461.

Simpson, M. L., Olejnik, S., Tam, A. Y.-W., \& Supattathum, S. (1994).
Elaborative verbal rehearsals and college students' cognitive performance. Journal of Educational Psychology, 86, 267-278.

Soler, M. J., \& Ruiz, J. C. (1996). The spontaneous use of memory aids at different levels of education. Applied Cognitive Psychology, 10, 41-51.

Sweller, J., van Merri nboer, J. J. G., \& PaAs, F. G. W. C. (1998). Cognitive architecture and instructional design. Educational Psychology Review, 10, 251-296.

van Hell, J. G., \& MAHN, A. C. (1997). Keyword mnemonics versus rote rehearsal: Learning of concrete and abstract foreign words by experienced and inexperienced learners. Language Learning, 47, 507-546.

\section{APPENDIX \\ English Translation of Beginning of the Passage "History of Sudan" and Its Division Into Idea Units}

One of the oldest kingdoms of the Black population was based in Sudan. / In the past, the climate was less arid than today, / shepherds were able to graze their herds in the savannah, / fishermen were able to find abundant fish in the lakes and rivers, / hunters found game in the woods, / and farmers cultivated the land in a rudimentary way. / These were purely subsistence activities and did not allow for the formation of a state, / which requires overproduction to maintain a ruling class, functionaries, and soldiers. / However, Sudan also had gold, / a commodity in demand by Mediterranean civilizations to mint money./

(Manuscript received May 3, 2001;

revision accepted for publication August 5, 2002.) 